\title{
Orthostatic Hypotension
}

National Institute of Neurological Disorders and Stroke (NINDS)

\section{Source}

National Institute of Neurological Disorders and Stroke (NINDS). Orthostatic

Hypotension Information Page.

Orthostatic hypotension is a sudden fall in blood pressure that occurs when a person assumes a standing position. It is due to a lesion of the baroreflex loop, which senses a change in blood pressure and adjusts heart rate and activates sympathetic nerve system fibers to cause the blood vessels to narrow and correct blood pressure. It may also be caused by hypovolemia (a decreased amount of blood in the body), resulting from the excessive use of diuretics, vasodilators, or other types of drugs, dehydration, or prolonged bed rest. The disorder may be associated with Addison's disease, diabetes, and certain neurological disorders including Multiple System Atrophy with Orthostatic Hypotension (formerly known as Shy-Drager syndrome), autonomic system neuropathies, and other dysautonomias. Symptoms, which generally occur after sudden standing, include dizziness, lightheadedness, blurred vision, and syncope (temporary loss of consciousness). 